Research Paper

\title{
Fluorofenidone Inhibits the Proliferation of Lung Adenocarcinoma Cells
}

\author{
Zheng-hao Deng1,2, Jie Meng³, Juan Tang4, Gao-yun Hu5, Li-jian Tao ${ }^{4,6 \bowtie ~}$ \\ 1. Department of pathology, Xiangya Hospital, Central South University, Changsha, Hunan, 410008, China \\ 2. Department of pathology, School of basic medicine, Central South University, Changsha, Hunan, 410078, China \\ 3. Department of pulmonary Medicine, XiangYa Hospital, Central South University, Changsha, Hunan, 410008, China \\ 4. Department of Nephropathy, XiangYa Hospital, Central South University, Changsha, Hunan, 410008, China \\ 5. Faculty of Pharmaceutical Sciences, Central South University, Changsha Hunan 410013, China \\ 6. State Key Laboratory of Medical Genetics of China, Changsha, Hunan 410078, China \\ $\square$ Corresponding author: Professor Lijian Tao, Email: taolj@csu.edu.cn \\ (c) Ivyspring International Publisher. This is an open access article distributed under the terms of the Creative Commons Attribution (CC BY-NC) license \\ (https://creativecommons.org/licenses/by-nc/4.0/). See http://ivyspring.com/terms for full terms and conditions.
}

Received: 2016.10.23; Accepted: 2017.04.08; Published: 2017.07.04

\begin{abstract}
Background: Lung carcinoma is the leading cause of malignant tumor related mortality in China in recent decades, and the development of new and effective therapies for patients with advanced lung carcinoma is needed. We recently found that fluorofenidone (FD), a newly developed pyridine compound, reduced the activation of Stat3 (Signal transducer and activator of transcription 3) in fibroblasts. Stat 3 plays a crucial role in the development of lung cancer and may represent a new therapeutic target. In this study, we examined the effect of FD on human lung adenocarcinoma cells in vivo and in vitro.

Methods: The effect of FD on the growth of lung cancer cells was measured with a CCK-8 assay, colony formation assay and xenograft tumor model. A flow cytometry analysis was performed to study cell cycle arrest and apoptosis. Western blotting and immunohistochemistry were used to observe the expression of Stat3. Changes in the expression of RNA induced by FD were assessed using gene chip and real-time RT-PCR assays.

Results: In vitro, FD inhibited the growth of lung adenocarcinoma A549 and SPC-A1 cells in a dose-dependent manner. After treatment with FD, the A549 and SPC-Al cells were arrested in the Gl phase, and apoptosis was induced. In vivo, this compound significantly inhibited the growth of tumors that were subcutaneously implanted in mice. Moreover, FD decreased Stat3 activity in lung cancer cells and xenograft tumor tissue, and microarray chip results showed that FD altered the gene expression profile of lung cancer cells. Specifically, NUPR 1, which plays a significant role in cancer development, was down-regulated by FD in lung cancer cells.

Conclusion: Our study supports the clinical evaluation of FD as a potential lung adenocarcinoma therapy.
\end{abstract}

Key words: Fluorofenidone, lung adenocarcinoma, Stat3, NUPR1.

\section{Introduction}

Malignant tumor is a major public health problem worldwide, and lung carcinoma is the most frequently diagnosed malignant tumor and it is responsible for over 1.6 million deaths each year worldwide [1]. In China, approximately 705,000 new cases of lung carcinoma are diagnosed per year [2], and it is often found at an advanced stage, which results in a poor prognosis. Non-small-cell lung cancer (NSCLC) is the most common histologic type of lung carcinoma [3], and the treatment for NSCLC based on platinum chemotherapy. But, clinical interventions have only minimally reduced deaths from lung cancer in the past four decades [4-5]. Despite recent improvements in early diagnosis and 
the evolution of novel therapeutic strategies, the prognosis for all stages of NSCLC continues to be poor, and the 5-year survival rate for all patients remains at less than $15 \%$ [1-2,6]. Thus, novel therapeutic approaches are needed for the treatment of lung cancer.

Fluorofenidone [1-(3-fluorophenyl)-5-methyl2-(1H)-pyridone, FD] is a new pyridine compound developed by our group. Our previous studies have reported that FD can attenuate fibrosis in the lung [7], kidney [8] and liver [9] via anti-inflammatory [10], antioxidative [11], and antifibrotic [12] activities. Recently, we found that FD reduced the activation of Stat3 (Signal transducer and activator of transcription 3) in fibroblast cells [13]. Notably, Stat3 plays a concernful role in carcinogenesis by regulating genes involved in anti-apoptosis, proliferation and angiogenesis [14]. Furthermore, More and more evidence indicates that Stat3 is constitutively activated in nearly $70 \%$ of tumors [15], and blocking Stat3 signaling in tumor cells inhibits tumor growth, angiogenesis, and metastasis [16]. Thus, Stat3 is recognized as a target for cancer therapy [17], but the ability of FD to reduce the activity of Stat 3 and inhibit the growth of cancer cells remains unknown.

Based on the important role of Stat3 in cancer pathogenesis and the effect of FD on the activity of Stat3 in fibroblasts, we investigated the ability of FD to inhibit the growth of lung cancer cells. Specifically, we herein examine the effects of FD on the growth and tumorigenicity of lung cancer cells.

\section{Materials and Methods}

\section{Reagents and antibodies}

FD (lot no.070501) was purchased from Sunshine Lake Pharma (Guangzhou, GD, CN). Cell culture reagents including RPMI1640, fetal bovine serum (FBS), and penicillin/streptomycin were obtained from Invitrogen (Carlsbad, CA, U.S.). Antibodies against cyclin D1, p-Stat3 (Y705) and NUPR1 were obtained from CST (Danvers, MA, USA). Anti $\beta$-actin antibody was purchased from Sigma-Aldrich Ltd (St. Louis, MO, USA). Horseradish peroxide (HRP)-conjugated secondary antibodies for Western blotting were procured from Jackson (West Grove, PA, USA), and secondary antibodies for immunohistochemistry were obtained from GBI (Mukilteo, WA, USA). The ECL kit was purchased from GE Healthcare (Buckinghamshire, U.K.), and the CCK-8 assay kit was obtained from Dojindo (Rockville, USA).

\section{Cell culture}

The human lung adenocarcinoma cell lines A549 and SPC-A1 were obtained from the cell bank of the
Type Culture Collection Chinese Academy of Sciences (Shanghai, China). The cells were cultured in RPMI 1640 medium supplemented with $10 \%$ FBS, $100 \mathrm{U} / \mathrm{ml}$ penicillin, $100 \mu \mathrm{g} / \mathrm{ml}$ streptomycin sulfate at $37^{\circ} \mathrm{C}$ and $5 \%$ CO2. Confluent cells were passaged with $0.25 \%$ trypsin.

\section{CCK-8 Assay}

A CCK-8 assay was performed as described previously [18]. Briefly, cells were plated in 96-well plates at a density of 2000 cells in a final volume of 100 $\mu \mathrm{l} /$ well. Samples of subconfluent cells were treated with various concentrations of $\mathrm{FD}(0,2$, and $4 \mathrm{mM})$ for 24, 48 and $72 \mathrm{~h}$, respectively. Cell growth was the assessed with a Cell Counting Kit-8 assay according to the manufacturer's protocol. All experiments were performed in triplicate.

\section{Colony Formation Assay}

A colony formation assay was performed as described previously [19]. Briefly, cells were trypsinized, and 1000 viable cells were subcultured in triplicate in six-well plates. The cells were treated with FD $(0,2$, and $4 \mathrm{mM})$ for $72 \mathrm{~h}$ and then allowed to adhere and colonize for 14 days without the drug. The medium was refreshed every 3 days. The cells were washed with PBS and fixed with $4 \%$ paraformaldehyde. The fixed cells were stained with freshly prepared Giemsa stain for 20 min and colonies were counted under a microscope. All experiments were performed in triplicate.

\section{Flow Cytometry Analysis}

Flow cytometry was used to analyze the cell cycle and apoptosis as previously described [20]. In brief, cells were treated with FD $(0,2$, and $4 \mathrm{mM})$ for $72 \mathrm{~h}$ and then harvested and fixed in 70\% ethanol at $4^{\circ} \mathrm{C}$ overnight. After staining with annexin $\mathrm{V}$ to analyze apoptosis or propidium iodide to analyze the cell cycle, the cells were examined with a BD FACSCalibur Flow Cytometer (BD Biosciences, San Jose, CA).

\section{Western Blot Analysis}

Whole-cell lysates were prepared for the western blot analysis as previously described [21]. Cell lysates containing $30 \mu \mathrm{g}$ of protein were separated on $10 \%$ SDS-polyacrylamide gels and transferred to PVDF membranes. The membranes were blocked with TBST buffer (10 mM Tris-HCl, pH 8.0, 0.15 M NaCl, and $0.05 \%$ Tween-20) containing 5\% FBS and incubated with a rabbit anti-human p-stat3 antibody (1:2000), cyclin D1 antibody $(1: 1000)$ or $\beta$-actin antibody $(1: 5000)$ at room temperature for $2 \mathrm{~h}$, followed by the addition of horseradish peroxidase-linked anti-goat IgG (1:2000). Blotted proteins were detected and 
quantified using an enhanced chemiluminescence detection system.

\section{Animal Studies}

The experimental protocol and the care of animals were under supervision of ethics committee of Central South University. Fifteen female BALB/c nude mice aged 4 to 6 weeks were purchased from Slac Laboratory Animal Co. Ltd. (Shanghai, China) and housed in individually ventilated cages. The mice were randomly divided into 3 groups ( 5 mice/group). Cultured A549 cells were harvested and resuspended in PBS at $1 \times 107$ cells $/ 0.2 \mathrm{ml}$. The suspension was subcutaneously injected into the left upper extremity of each nude mouse, and treatment with FD was initiated 7 days after inoculation with the cells. The mice were treated daily with intragastrically administered $0.5 \%$ carboxymethyl cellulose sodium or fluorofenidone $(500 \mathrm{mg} / \mathrm{kg} /$ day or $1000 \mathrm{mg} / \mathrm{kg} /$ day) for the next 4 weeks until they were euthanized. The tumor volume (TV) was calculated according to the following formula: TV $(\mathrm{mm} 3)=\mathrm{d} 2 \times \mathrm{D} / 2$, where $\mathrm{d}$ and $\mathrm{D}$ are the shortest and longest diameters, respectively. The mice were sacrificed 5 weeks after cell implantation, and the tumors were extracted, weighed, fixed in 10\% formalin, embedded in paraffin, and cut into $4 \mu \mathrm{m}$-thick sections for further study.

\section{Immunohistochemistry}

The immunohistochemistry assay was performed as described previously [22]. Briefly, sections were incubated at $60^{\circ} \mathrm{C}$ for $2 \mathrm{~h}$ in turpentine and deparaffinized, dried, and rehydrated in a decreasing ethanol: distilled water gradient series. The sections were then incubated for $30 \mathrm{~min}$ in $0.3 \%$ hydrogen peroxide in $0.01 \mathrm{M}$ phosphate-buffered saline (PBS) to inactivate endogenous peroxidases. Subsequently, the sections were incubated for $20 \mathrm{~min}$ in citrate buffer $\left(\mathrm{pH} \mathrm{6.0)}\right.$ at $95^{\circ} \mathrm{C}$ for antigen retrieval, blocked with 5\% normal goat serum in $0.01 \mathrm{M}$ PBS for $15 \mathrm{~min}$ at $37^{\circ} \mathrm{C}$, and incubated with primary antibodies overnight at $4^{\circ} \mathrm{C}$ in a humidified chamber. The following primary antibodies were used: a rabbit anti-p-Stat3 (Y705) antibody (1:500) and a rabbit anti-cyclind1 (1:500). After washing, the samples were incubated with a biotinylated secondary antibody and a streptavidin-horseradish peroxidase (HRP) avidin working solution. Immunolabeling was visualized using a DAB substrate kit according to the manufacturer's recommendations.

\section{Gene Microarray}

The gene expression patterns of lung cancer cells treated with FD $(4 \mathrm{mM}, 72 \mathrm{~h})$ were detected with a PrimeView $^{\mathrm{TM}}$ Human Gene Expression Array
(Affymetrix, CA, USA). The cells were lysed in TRIzol (Invitrogen, CA, USA) to extract RNA, and microarray assays were then performed by CapitalBio Corporation (Beijing, China).

\section{RNA extraction and real-time RT-PCR}

RNA extraction and real-time RT-PCR were performed as previously described [23]. The primers were synthesized by Bio Basic Inc (Shanghai, China). The primer against NUPR1 was 5'-CTGTGT TTACTGGGCTGGATT-3' (forward) and 5'-AAGGCT GTGTCTCCTCCTCA-3' (reverse). The primer against $\beta$-actin was 5'-CCTTCCTGGGCATGGAGTCCT-3' (forward) and 5'-GGAGCAATGATCTTGATCTT-3' (reverse). Real-time RT-PCR was performed on an ABI Model 7500 Sequence Detector (Applied Biosystems, Foster City, CA) with a TaKaRa SYBR green real-time PCR kit (Takara, Dalian, China). The quantity of mRNA was calculated based on the cycle threshold (CT) values, and the relative abundance of NUPR1 mRNA was standardized to that of $\beta$-actin mRNA.

\section{Statistical analysis}

The SPSS 17.0 statistical software package (SPSS Inc., Chicago, IL, USA) was used to perform statistical analyses. Student's t-test was used to perform comparisons between two groups. Pearson's correlation coefficients were used to calculate bivariate correlations between study variables. The data represent the mean \pm S.D. A P-value $<0.05$ was considered significant.

\section{Results}

\section{FD inhibited growth of lung adenocarcinoma cells.}

To investigate the effects of FD on the growth of human lung adenocarcinoma cells, a CCK-8 assay and colony formation assay were performed. FD inhibited the growth of A549 and SPC-A1 cells over 48 and $72 \mathrm{~h}$ of continuous exposure, as assessed by the CCK-8 assay (Figure 1). Specifically, the suppression rates were $42.14 \pm 0.17 \%$ at $48 \mathrm{~h}$ and $48.73 \pm 0.32 \%$ at $72 \mathrm{~h}$ for A549 cells treated with $4 \mu \mathrm{m} \mathrm{FD,} \mathrm{and} \mathrm{the} \mathrm{suppression}$ rates were $35.64 \pm 0.48 \%$ at $48 \mathrm{~h}$ and $45.27 \pm 0.19 \%$ at $72 \mathrm{~h}$ for SPC-A1 cells treated with $4 \mu \mathrm{m}$ FD. We analyzed the ability of plated A549 and SPC-A1 cells to form colonies because anchorage-independent growth often correlates with tumorigenicity. The respective plating efficiencies of the cells treated with 0,2 , and 4 mM FD were $12.5 \pm 1.5 \%, 10.9 \pm 2.1 \%$ and $3.9 \pm 0.6 \%$ for the A549 cells and $13.4 \pm 2.5 \%, 9.8 \pm 3.3 \%$ and $5.1 \pm 0.9 \%$ for the SPC-A1 cells (Fig. 2). FD decreased colony formation only at a dose of $4 \mathrm{mM}$ compared with the other two groups $(\mathrm{P}<0.05)$. Therefore, it inhibited lung adenocarcinoma cell growth. 
A

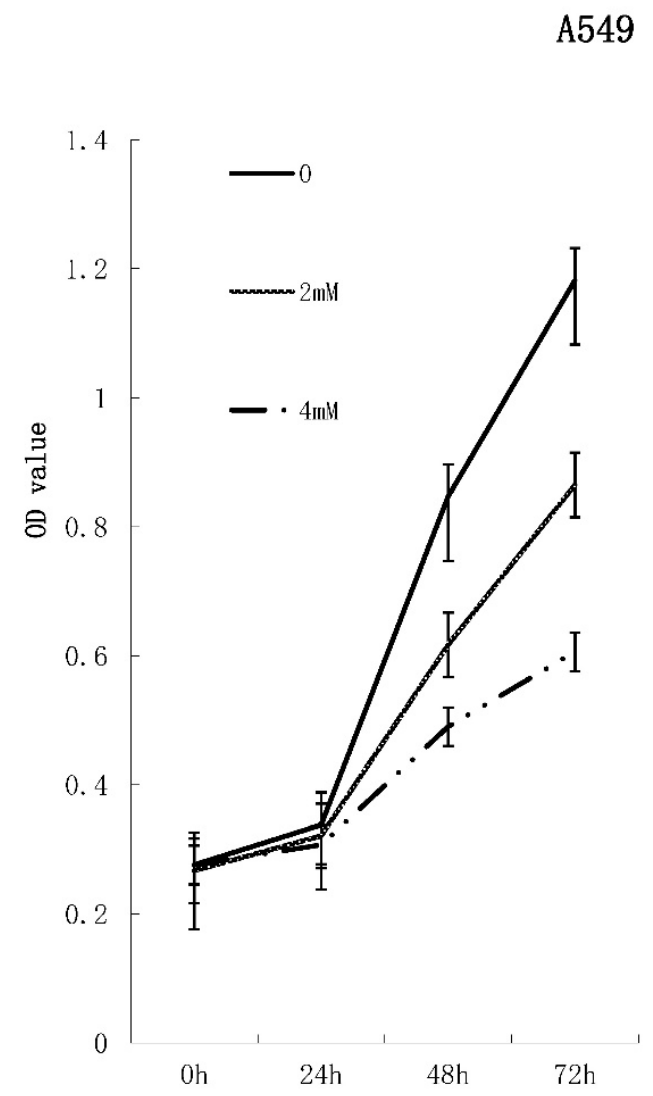

B

$\mathrm{SPC}-\mathrm{A} 1$

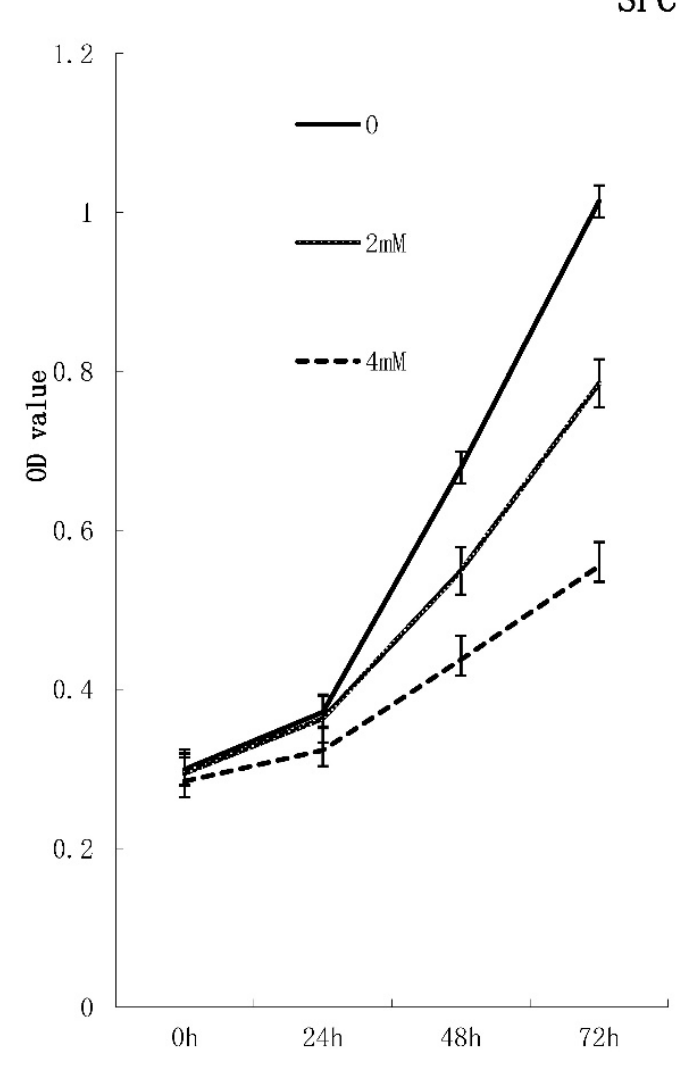

Figure 1. FD inhibits the growth of lung adenocarcinoma cells in vitro. A549 cells and SPC-Alcells were treated with FD at the indicated concentration for $72 \mathrm{~h}$. Cell viability was measured with a CCK-8 assay. FD inhibited the growth of A549 (Fig. 1A) and SPC-A1 (Fig. 1B) cells over 48 and $72 \mathrm{~h}$ of continuous exposure. Experiments were performed in triplicate.
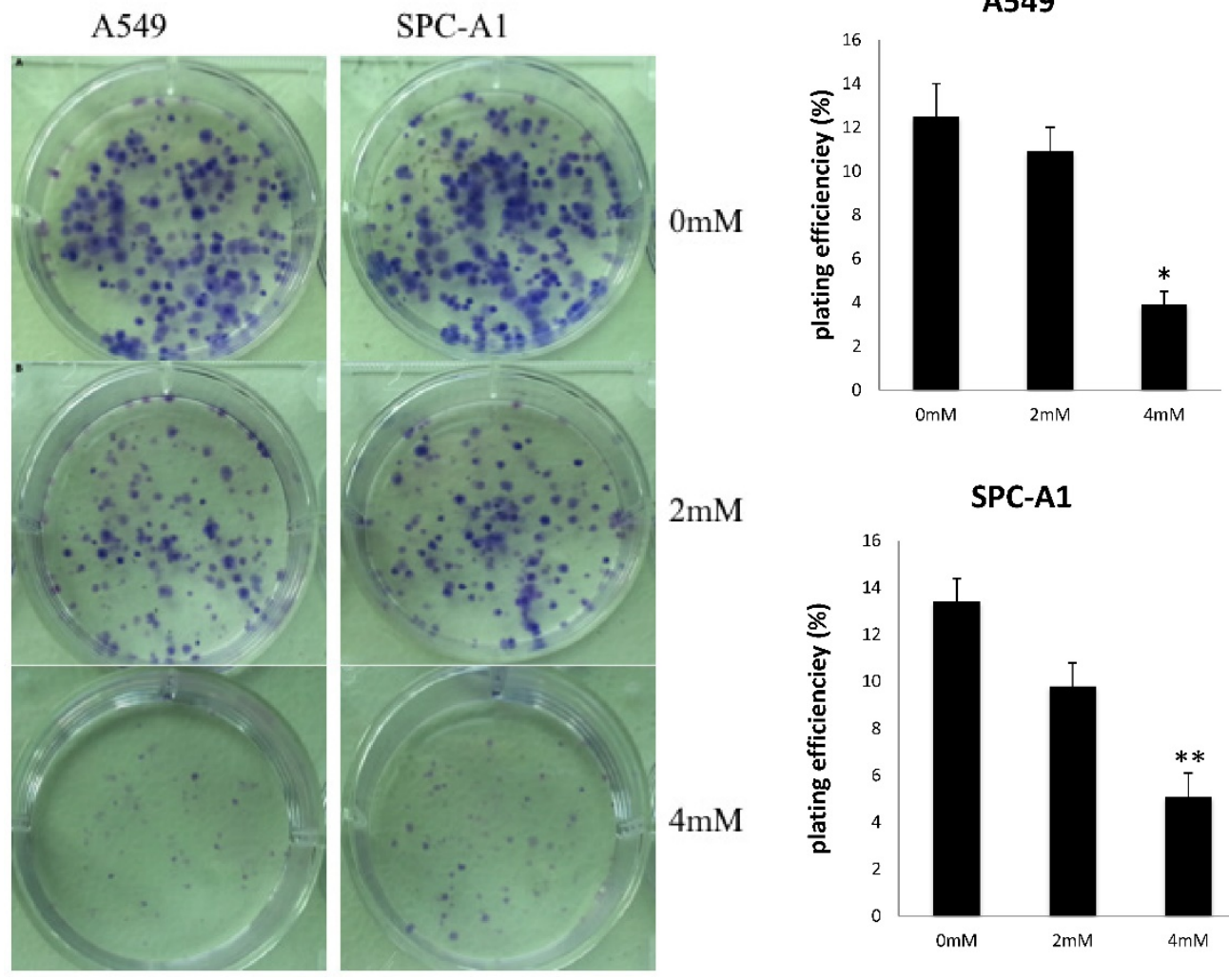

$2 \mathrm{mM}$

SPC-A1
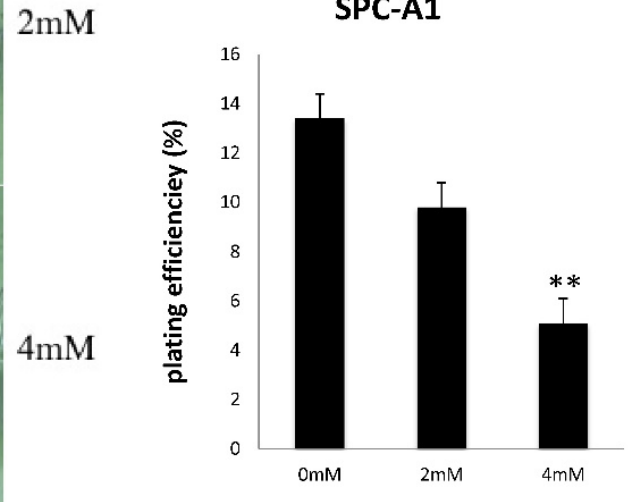

Figure 2. FD decreased the colonies formed by A549 and SPC-A1 cells in a dose-dependent. Experiments were performed in triplicate. (Compared with the control group,*, **, $\mathrm{P}<0.05$ ) 


\section{FD induced lung adenocarcinoma cell G 1 arrest and apoptosis.}

To investigate the effects of FD on the cell cycle and apoptosis of human lung adenocarcinoma cells, cells were assessed by flow cytometry after treatment with different concentrations of $\mathrm{FD}(0,2$, and $4 \mathrm{mM})$ for $72 \mathrm{~h}$. As shown in Fig. 3, FD induced cell cycle transition to the $\mathrm{G} 1$ phase $(\mathrm{P}<0.05)$. The apoptosis rate of the cells treated with 0,2 , and $4 \mathrm{mM}$ FD were $1.4 \pm 0.1 \%, 2.9 \pm 0.2 \%$ and $4.0 \pm 0.3 \%$ for the A549 cells and $3.4 \pm 0.6 \%, 6.8 \pm 0.3 \%$ and $10.7 \pm 1 \%$ for the SPC-A1 cells. Hence, FD increased the number of apoptotic cells compared with the respective controls $(\mathrm{P}<0.05)$.

\section{FD inhibited lung adenocarcinoma cell growth in vivo.}

We evaluated the effects of FD in vivo in a xenograft tumor model by subcutaneously inoculating nude mice with A549 cells. Seven days after tumor cell injection, the mice were administered daily intragastric injections of FD (500 and 1000 $\mathrm{mg} / \mathrm{kg}$ body weight) for 4 weeks. The tumors appeared at nearly the same time in the three groups. At the end of the study, all tumors were removed and dissociated, and their weights and volumes were measured. The mean tumor weights of the three groups (treated with FD 0,500 and $1000 \mathrm{mg} / \mathrm{kg}$ body weight) were $0.625 \pm 0.1 \mathrm{~g}, 0.68 \pm 0.08 \mathrm{~g}$ and $0.32 \pm 0.04 \mathrm{~g}$, respectively. The mean tumor volumes of the three groups (treated with FD 0,500 and $1000 \mathrm{mg} / \mathrm{kg}$ body weight) were $589.6 \pm 97.7 \mathrm{~mm}^{3}, 632.6 \pm 56.7 \mathrm{~mm}^{3}$ and $265.9 \pm 56.7 \mathrm{~mm}^{3}$, respectively. Consistent with the in vitro results, FD decreased the growth of A549 cells. FD administration was effective in inhibiting tumor growth in vivo throughout the course of treatment, resulting in decreases in tumor size and weight at a dose of $1000 \mathrm{mg} / \mathrm{kg}$ body weight $(\mathrm{P}<0.05)$ (Figure 4). It indicated that FD reduced tumor volume and the growth of A549 cells in vivo.

\section{FD decreased Stat3 activity in lung adenocarcinoma cells.}

Western blotting data showed that p-stat 3 and cyclin D1 expression were significantly inhibited in A549 and SPC-A1 cells treated with $4 \mathrm{mM}$ FD compared with the control group and cells treated with $2 \mathrm{mM}$ FD. Furthermore, immunohistochemistry assay showed that both p-Stat3 and cyclind1 expression were inhibited in xenograft tumors treated with $1000 \mathrm{mg} / \mathrm{kg}$ body weight compared with the other two groups $(\mathrm{P}<0.05)$ (Fig. 5).

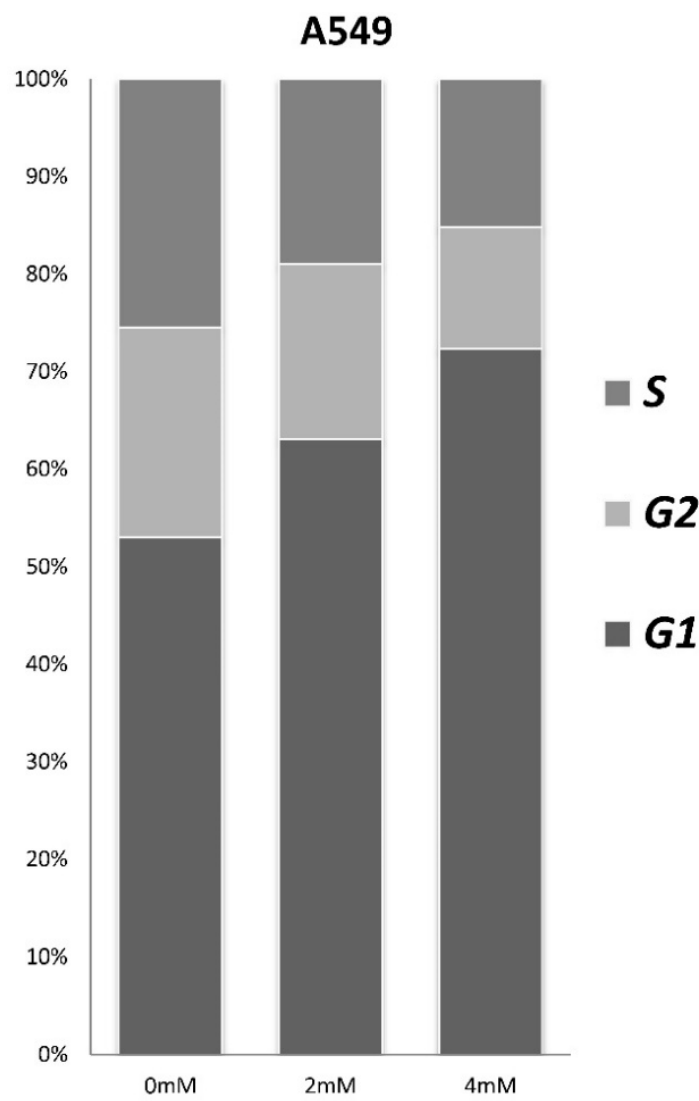

\section{SPC-A1}

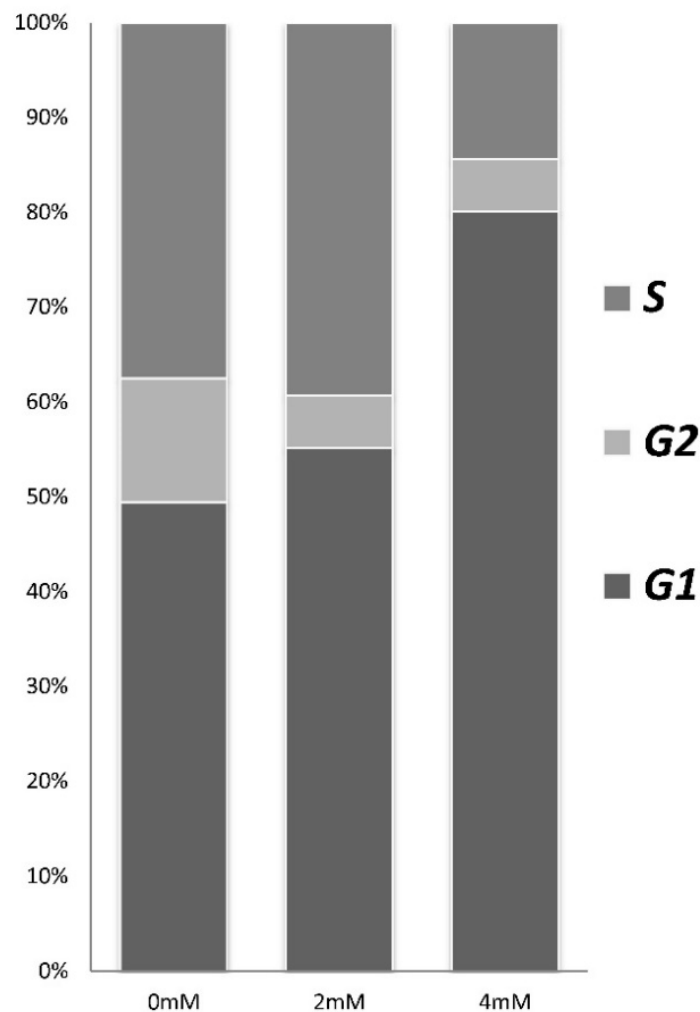

Figure 3. FD induces Gl arrest in lung adenocarcinoma cells. Cells were stained with annexin-V-FITC (20 mg/ml) and PI (20 mg/l) following treatment with or without FD for $72 \mathrm{~h}$. The cell cycle was analyzed using flow cytometry. Experiments were performed in triplicate. 


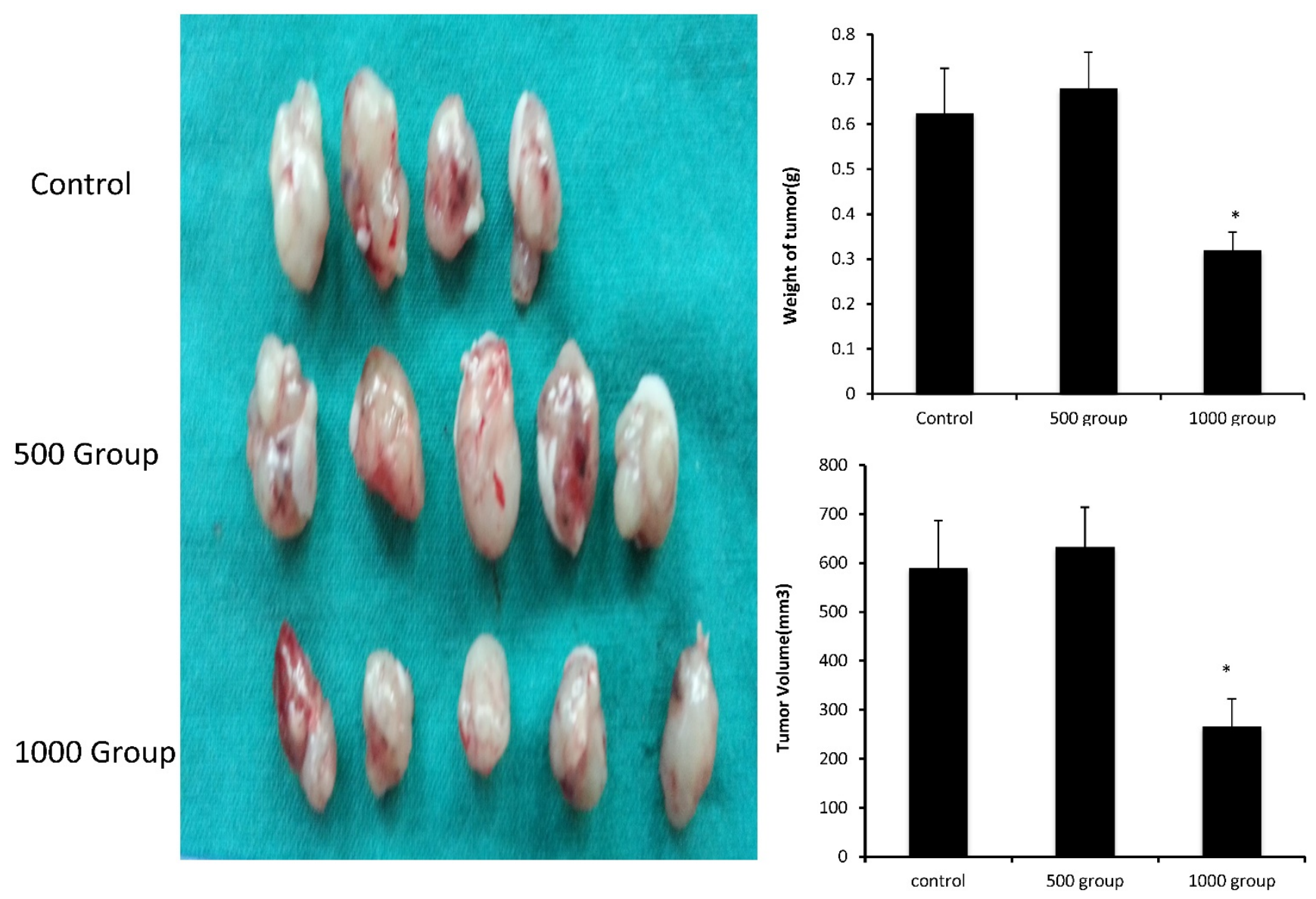

Figure 4. FD inhibits lung adenocarcinoma A549 cell tumor growth in vivo. Dissected tumors were photographed, and the weights and volumes of tumors from FD-treated mice $(0,500 \mathrm{mg} / \mathrm{kg} /$ day and $1000 \mathrm{mg} / \mathrm{kg} /$ day) were measured. (Compared with the control group, *, **, $\mathrm{P}<0.05)$

\section{Effects of FD on gene expression pattern on lung cancer cells.}

The effect of FD on gene expression in A549 and SPC-A1 cells was assessed using Affymetrix gene microarrays. Gene expression profile was examined in cells treated with 0 or $4 \mathrm{mM}$ FD for $72 \mathrm{~h}$. The differentially expressed genes were identified including up-regulated 36 genes and down-regulated 40 genes in both A549 and SPC-A1 cells (compared with the control group, expression ratio showing a greater than 2-fold or a less than 0.5-fold was identified). These genes are involved in Wnt signaling, PPAR signaling, MAPK signaling and ErbB signaling (data not shown). Therefore, FD may have its anti-tumor effect on lung carcinoma cells by changing the expression of these genes. Among these differentially expressed genes, NUPR1 expression was increased more than 5-fold in A549 and SPC-A1 cells. Western blotting and real time-PCR results showed that the mRNA and protein expression levels of NUPR1 were markedly reduced in FD-treated lung carcinoma cells, which is consistent with the gene array analysis (Fig. 6).

\section{Discussion}

In this study, we first described the effects of FD on lung cancer cells in vitro and in vivo. Specifically, we found that FD inhibited the growth of lung adenocarcinoma A549 and SPC-A1 cells using a CCK-8 assay and colony formation assay. Moreover, treatment with FD arrested these cells in the G1-phase and induced apoptosis. Furthermore, FD significantly inhibited the growth of tumors subcutaneously implanted in mice. We also found that FD suppressed the expression of p-STAT3 in lung adenocarcinoma A549 and SPC-A1 cells, and FD have markedly affected the mRNA expression pattern of these cells.

The same effects have been reported in malignant glioma [24] and pancreatic cancer cells [25] for pirfenidone, whose chemical structure is similar to that of FD. However, pirfenidone reportedly inhibited pancreatic cancer growth by suppressing desmoplasia via the regulation of pancreatic stellate cells [25]. In our study, the mice were orally administered 500 and $1000 \mathrm{mg} / \mathrm{kg} /$ day FD. Growth inhibition was only observed in the high-dose group without obvious side effects. Thus, FD may inhibit the growth of lung adenocarcinoma cells in a dose-dependent manner. 


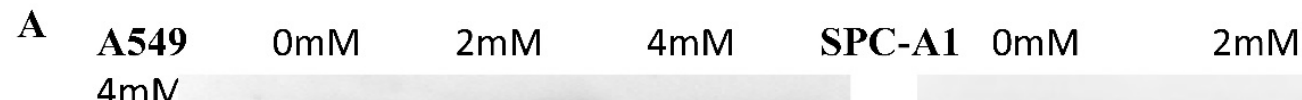

$4 \mathrm{mN}$

p-Stat3

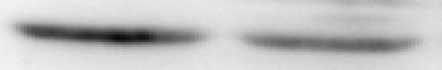

Cyclind1
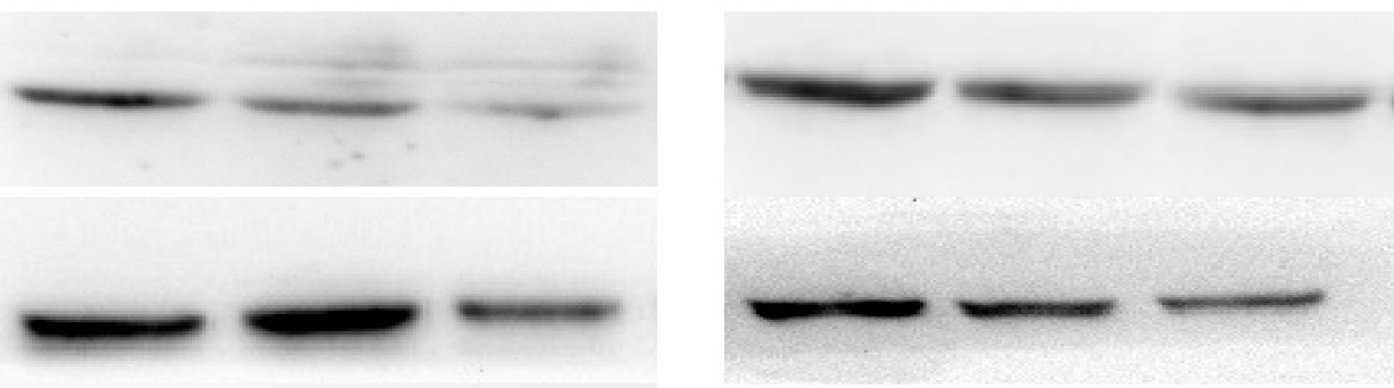

$\beta$-actin
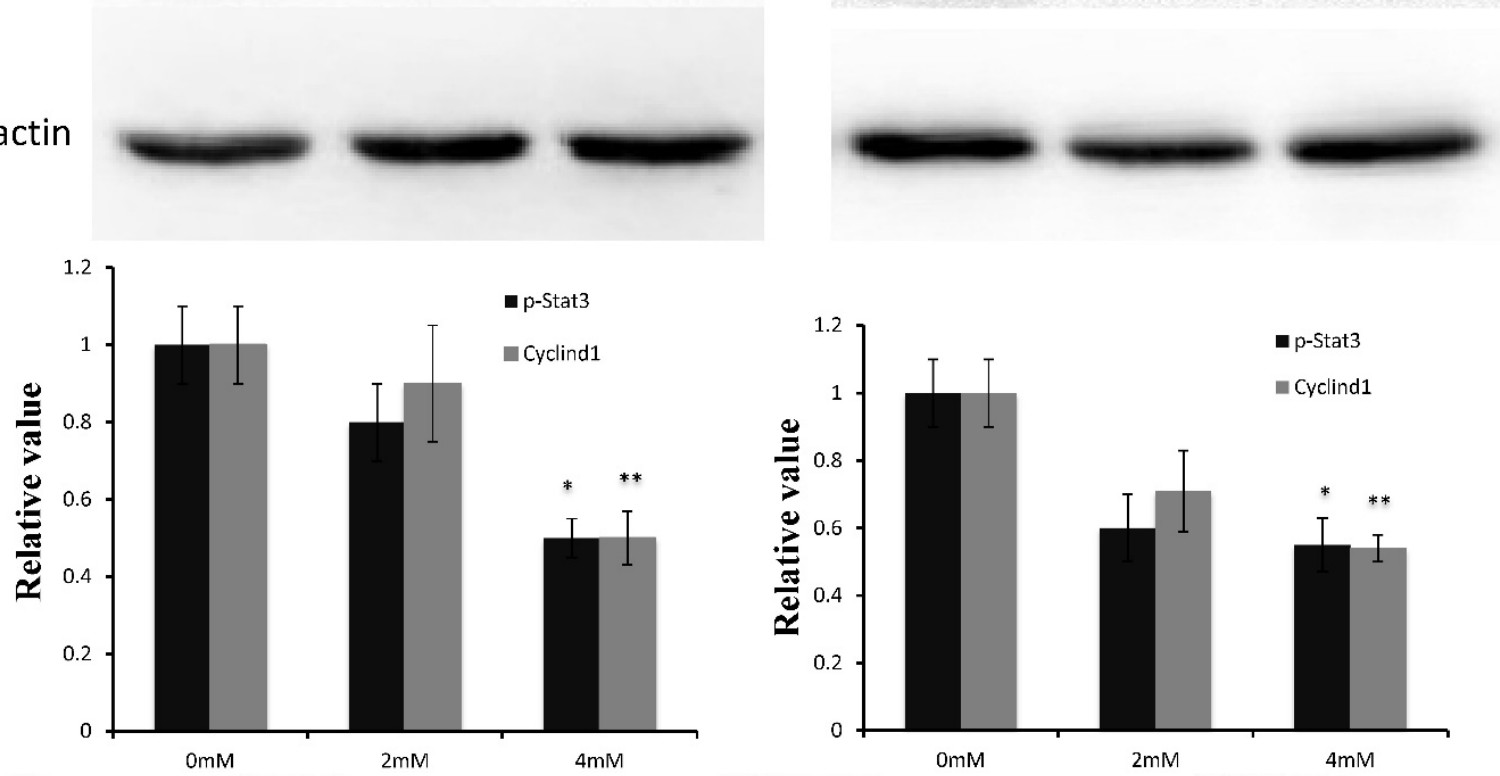

B

Control
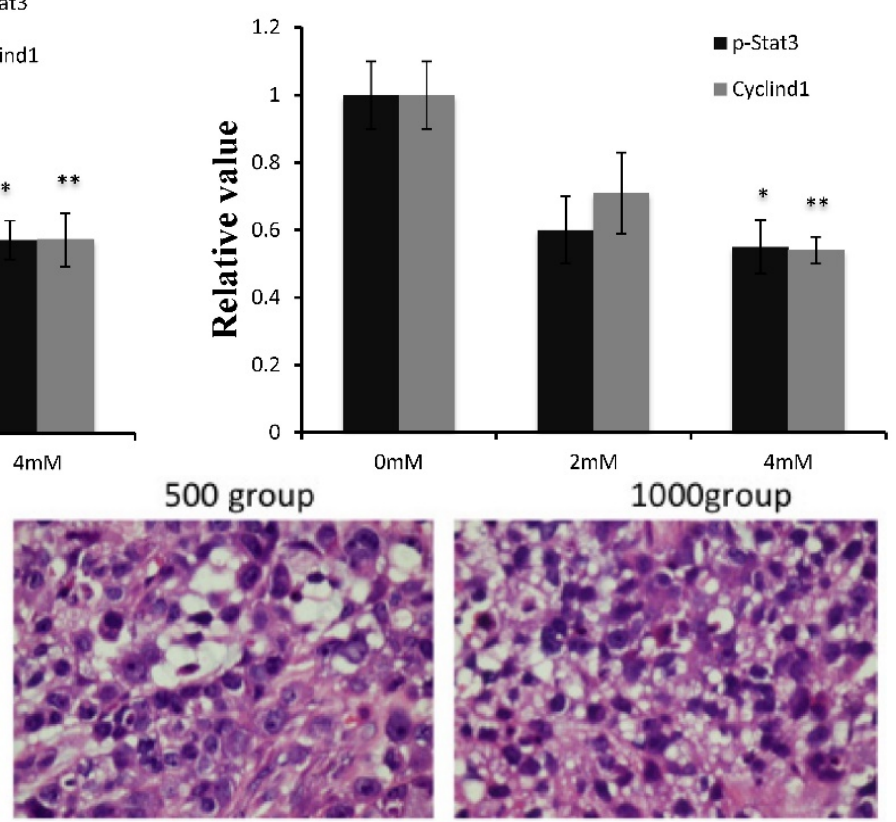

HE
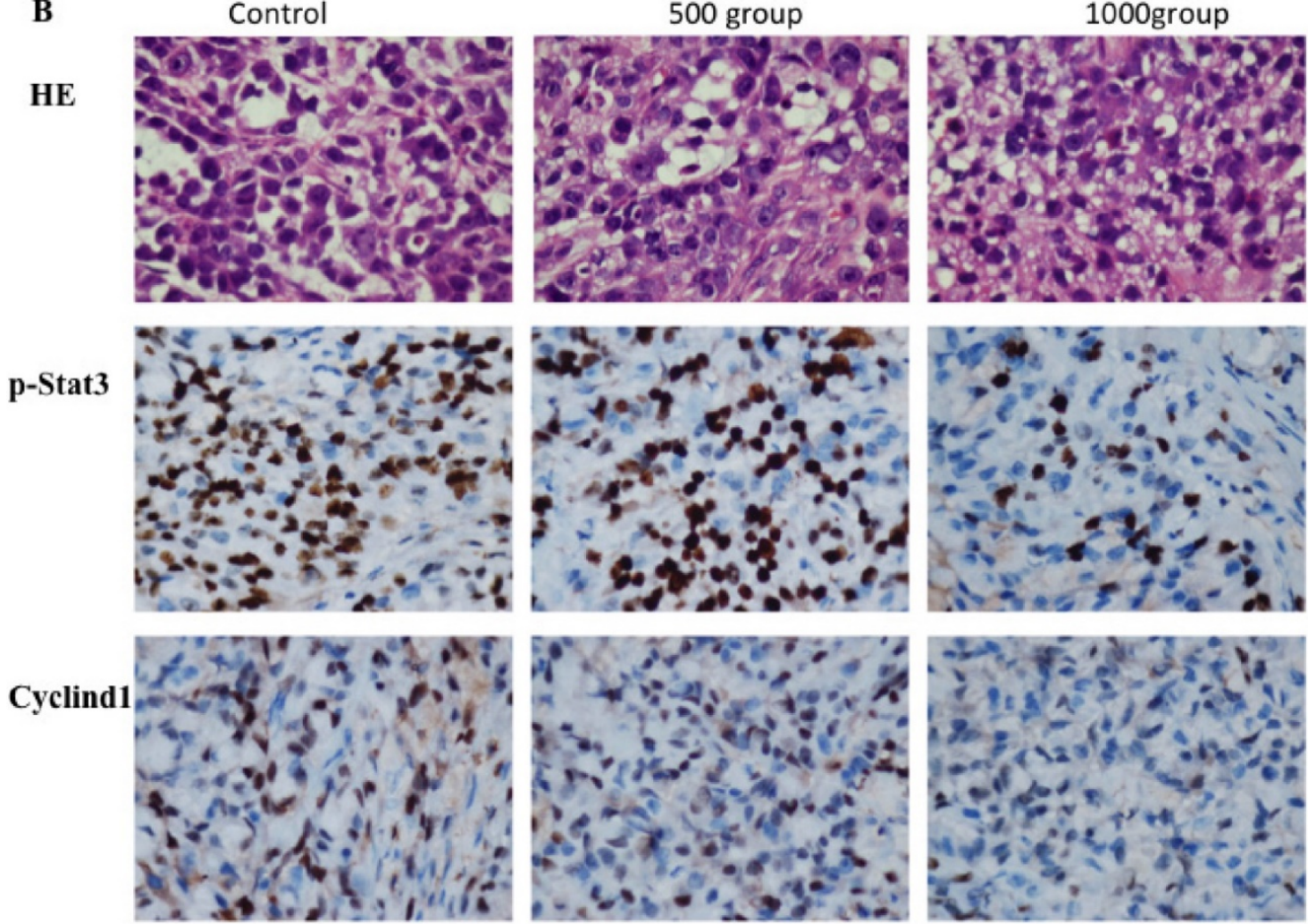

Figure 5. FD decreased Stat3 activity. (1) Western blotting results showed that FD treatment repressed the expression of p-Stat3 and cyclin D1 in lung adenocarcinoma A549 and SPC-A1 cells (Fig. 5A). (2) Representative images of the immunohistochemistry analysis of p-Stat3 and cyclin D1 in xenograft tumors ( $\times 400)$ (Fig. 5B). (Compared with the control group, *, **, $\mathrm{P}<0.05)$ 


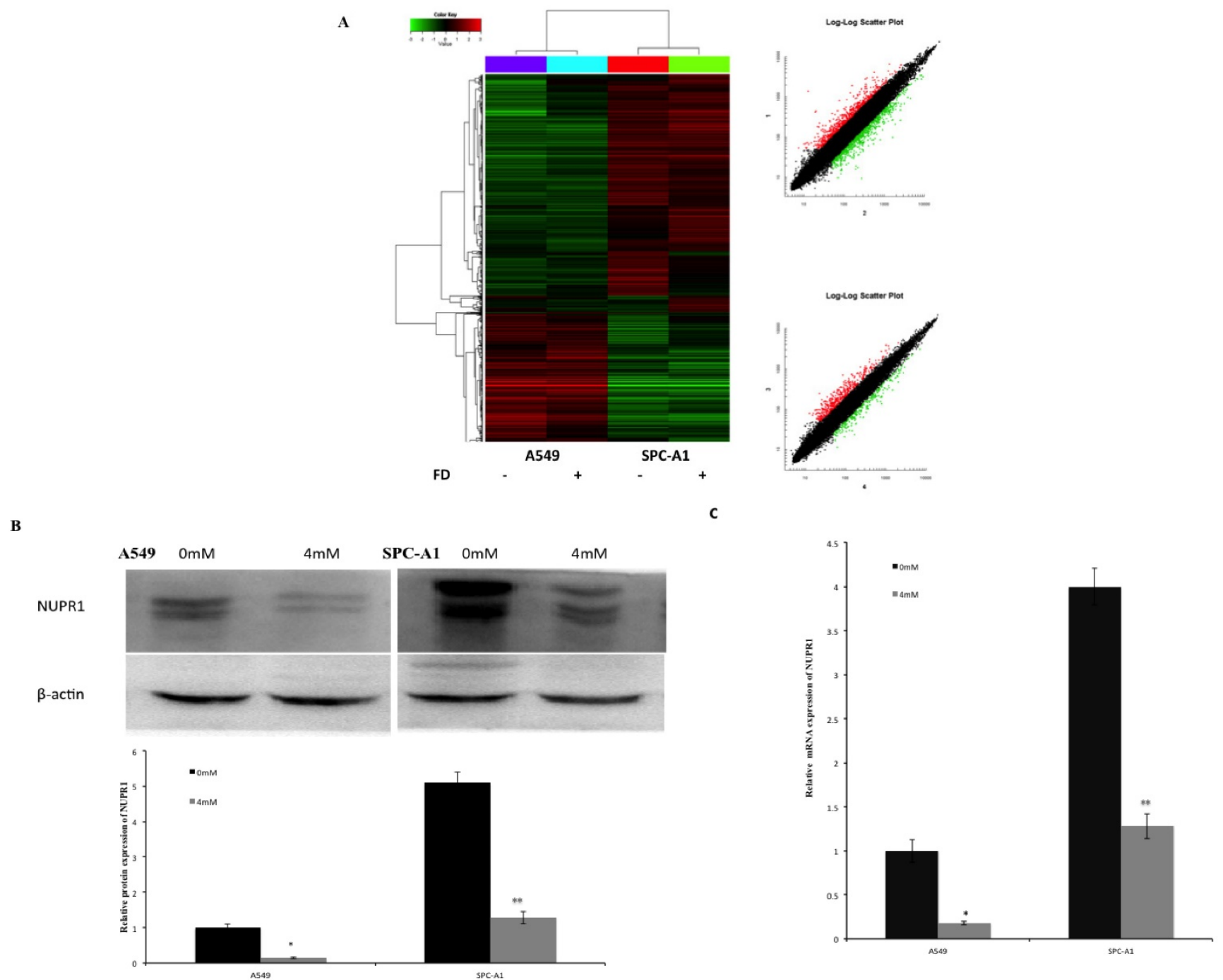

Figure 6. Effects of FD on the gene expression pattern of lung cancer cells. (1) Cluster map and scatter plot of changes in gene expression levels in lung cancer cells (Fig. 6A). (2) FD decreased the protein expression of NUPR1 in lung cancer cells, as determined by Western blotting (Fig. 6B). (3) FD decreased the mRNA expression of NUPR1 in lung cancer cells, as determined by real-time qPCR (Fig. 6C). (Compared with the control group, *, **, P<0.05)

In this study, we also found that FD inhibited the expression of p-STAT3 in lung cancer cells. STAT3 is a well-known member of the STAT family of signal responsive transcription factors, which consists of seven members encoded by distinct genes [26]. The phosphorylation of a critical tyrosine residue (Tyr705) activates STAT3 [27], and phosphorylated STAT3 forms a homodimer by binding at the $\mathrm{SH} 2$ domain and is translocated to the nucleus to promote the transcription of target genes [28]. Specifically, STAT3 regulates cyclin D1, c-Myc, Bcl-XL, and p53, thereby mediating cellular proliferation and survival [29]. Moreover, STAT3 is persistently activated in $22 \sim 65 \%$ of non-small cell lung cancers (NSCLCs) and plays an important role as a signaling mediator in malignant diseases [30-32]. An increasing body of evidence has shown that STAT3 inhibitors exert anticancer and antiangiogenic effects in vitro and in vivo [33]. These results indicate that FD may serve as an anti-cancer agent by inhibiting the phosphorylation of STAT3.

Our previous studies indicated that FD is a multifunctional small molecule with anti-inflammatory, antioxidative and anti-apoptotic effects, and it can inhibit the activation and proliferation of myofibroblasts, promote the degradation of extracellular matrix and regulate the cellular signal transmission [34]. However, the exact mechanisms underlying these effects remain unknown. To further explore the mechanism of action of FD in lung cancer cells, we observed the changes in gene expression by A549 and SPC-A1 after treatment with FD. Our results showed that FD markedly changed the gene expression patterns of these cells. Specifically, FD significantly decreased the expression of NUPR1 (Nuclear protein 1) in both A549 and SPC-A1 cells.

NUPR1, also named P8 or com1 (candidate of metastasis-1), is a small, highly basic and loosely 
folded protein. Structurally, Nupr1 is related to the high-mobility group of transcriptional regulators [35], and its expression can be induced by several stressors, including LPS [36] and CCL4 [37]. Many studies have shown that NUPR1, which is frequently upregulated in several cancers, plays an important role in cancer development and progression [38]. For example, NUPR1 is essential for the survival of pancreatic ductal adenocarcinoma cells exposed to stress [39]. Similar to our results, $X$. Guo reported that the knockdown of NUPR1 significantly inhibited the proliferation and colony formation of lung cancer H1299 cells and suppressed tumor growth in vivo [40]. Moreover, Roxane reported that the expression of NUPR1 was activated by TGF $\beta$ at the transcriptional level [41], and our previous studies showed that FD attenuated TGF- $\beta 1$-related signaling [13]. Thus, FD may have decreased the expression of NUPR1 by lung cancer cells via TGF- $\beta$ signaling, but this mechanism requires further investigation.

Overall, the present study revealed that FD inhibits the growth of lung adenocarcinoma A549 and SPC-A1 cells in vitro and in vivo. Thus, FD may represent a new treatment strategy for lung cancer, but additional studies are needed to clarify the mechanisms of action of FD in cancer cells.

\section{Abbreviations}

FD: Fluorofenidone; Stat3: Signal transducer and activator of transcription 3; NSCLC: Non-small-cell lung cancer; CCK-8: Cell Counting Kit-8.

\section{Acknowledgement}

This work was supported by National Natural Science Foundation of China (81470255 and 81273575).

\section{Authors' contributions}

Zhenghao Deng, Jie Meng, and Juan Tang collected and analyzed the data, drafted the manuscript, and conceived the main idea of the study. Gao-yun $\mathrm{Hu}$ and Lijian Tao participated in the acquisition of data and the design and intellectual conception of the study. All authors performed the statistical analysis and approved the final version of the manuscript.

\section{Ethics approval and consent to participate}

The experimental protocol and the care of animals accorded with the licenses held by the Central South University.

\section{Competing Interests}

The authors declare no conflicts of interest.

\section{References}

[1] Ferlay J, Shin HR, Bray F et al. Estimates of worldwide burden of cancer in 2008: GLOBOCAN 2008. Int J Cancer. 2010,127: 2893-2917.
[2] Lindsey A. Torre, Freddie Bray et al. Global Cancer Statistics, 2012, CA CANCER J CLIN, 2015,65: 87-108.

[3] Aisner DL, Marshall CB. Molecular pathology of non-small cell lung cancer: a practical guide. Am J Clin Pathol. 2012,138: 332-346.

[4] Spira A, Ettinger DS. Multidisciplinary management of lung cancer. N Engl J Med. 2004,350:379-392.

[5] Herbst RS, Heymach JV, Lippman SM. Lung cancer. N Eagl J Med. 2008,359: 1367-1380.

[6] Zarogoulidis P, Darwiche K, Browning RF et al. Molecular Targeted Drugs and Biomarkers in NSCLC, the Evolving Role of Individualized Therapy. J Cancer. 2013, 4: 736-754

[7] Meng J, Zou Y, Hu C et al. Fluorofenidone attenuates bleomycin-induced pulmonary inflammation and fibrosis in mice via restoring caveolin 1 expression and inhibiting mitogen-activated protein kinase signaling pathway. Shock. 2012, 38:567-73.

[8] Yuan Q, Wang R, Peng Y et al. Fluorofenidone attenuates tubulointerstitial fibrosis by inhibiting TGF- $\beta(1)$-induced fibroblast activation. Am J Nephrol. 2011,34: 181-94.

[9] Peng $\mathrm{Y}$, Yang $\mathrm{H}$, Wang $\mathrm{N}$ et al. Fluorofenidone attenuates hepatic fibrosis by suppressing the proliferation and activation of hepatic stellate cells. Am J Physiol Gastrointest Liver Physiol. 2014,306:253-63.

[10] Huang L, Zhang F, Tang Y et al. Fluorofenidone attenuates inflammation by inhibiting the NF-KB pathway. Am J Med Sci. 2014, 348:75-80.

[11] Qin J, Xie YY, Huang L et al. Fluorofenidone inhibits nicotinamide adeninedinucleotide phosphate oxidase via PI3K/Akt pathway in the pathogenesis of renal interstitial fibrosis. Nephrology (Carlton).2013, 18: 690-9.

[12] Peng $Y$, Yang $H$, Zhu $T$ et al. The antihepatic fibrotic effects of fluorofenidone via MAPK signalling pathways. Eur J Clin Invest. 2013, 43: 358-68.

[13] Tang J, Liu CY, Lu MM et al. Fluorofenidone protects against renal fibrosis by inhibiting STAT3 tyrosine phosphorylation. Mol Cell Biochem, 2015, 407(1-2): 77-87.

[14] Wake MS, Watson CJ. STAT3 the oncogene - still eluding therapy? FEBS J. 2015, 282(14): 2600-11.

[15] Siveen KS, Sikka S, Surana R, et al. Targeting the STAT3 signaling pathway in cancer: role of synthetic and natural inhibitors. Biochim Biophys Acta. 2014, 1845(2): 136-54.

[16] Wang X, Crowe PJ, Goldstein D, et al. STAT3 inhibition, a novel approach to enhancing targeted therapy in human cancers. Int J Oncol. 2012, 41(4): 1181-91.

[17] Muhammad Furqan, Akintunde Akinleye, Nikhil Mukhi. STAT inhibitors for cancer therapy. Journal of Hematology \& Oncology. 2013, 6: 90.

[18] Wang LL, Hu RC, Dai AG et al. Bevacizumab induces A549 cell apoptosis through the mechanism of endoplasmic reticulum stress in vitro. Int J Clin Exp Pathol. 2015, 8(5): 5291-5299.

[19] Li Q, Lu XH, Wang CD et al. Antiproliferative and apoptosis-inducing activity of schisandrin B against human glioma cells. Cancer Cell Int. 2015, 15(1): 12.

[20] Kim MO, Lee MH, Oi N et al. [6]-shogaol inhibits growth and induces apoptosis of non-small cell lung cancer cells by directly regulating Akt1/2. Carcinogenesis. 2014, 35(3): 683-91.

[21] Liu J, Song C, Xiao Q et al. Fluorofenidone attenuates TGF- $\beta 1$-induced lung fibroblast activation via restoring the expression of caveolin-1. Shock. 2015, 43(2): 201-207.

[22] $\mathrm{Hu}$ C, Lv H, Pan G et al. The expression of ADAM23 and its correlation with promoter methylation in non-small-cell lung carcinoma. Int J Exp Pathol. 2011, 92(5): 333-9.

[23] Xiong X, Mei W, Xie $Y$ et al. Fluorofenidone offers improved renoprotection at early interventions during the course of diabetic nephropathy in $\mathrm{db} / \mathrm{db}$ mice via multiple pathways. PLoS One. 2014, 9(10): e111242.

[24] Burghardt I, Tritschler F, Opitz CA, et al. Pirfenidone inhibits TGF-beta expression in malignant glioma cells. Biochem Biophys Res Commun. 2007, 354: 542-7.

[25] Kozono S, Ohuchida K, Eguchi D et al. Pirfenidone inhibits pancreatic cancer desmoplasia by regulating stellate cells. Cancer Res. 2013, 73:2345-56.

[26] Turkson J. STAT proteins as novel targets for cancer drug discovery. Expert Opin Ther Targets. 2004, 8(5): 409-22.

[27] Dutta P, Sabri N, Li J et al. Role of STAT3 in lung cancer. JAKSTAT. 2015, 3(4): e999503.

[28] Giraud AS, Menheniott TR, Judd LM. Targeting STAT3 in gastric cancer. Expert Opin Ther Targets. 2012, 16(9): 889-901.

[29] Hai-Feng Zhang, Raymond Lai. STAT3 in Cancer-Friend or Foe? Cancers. 2014, 6: 1408-1440.

[30] Zimmer S, Kahl P, Buhl TM et al. Epidermal growth factor receptor mutations in non-small cell lung cancer influence downstream Akt, MAPK and Stat3 signaling. J Cancer Res Clin Oncol. 2009, 135:723-30.

[31] Looyenga BD, Hutchings D, Cherni I, et al. STAT3 is activated by JAK2 independent of key oncogenic driver mutations in non-small cell lung carcinoma. PLoS One. 2012, 7:e30820.

[32] Jiang R, Jin Z, Liu Z et al. Correlation of activated STAT3 expression with clinicopathologic features in lung adenocarcinoma and squamous cell carcinoma. Mol.Diagn.Ther. 2011,15: 347-52.

[33] Daijiro Harada, Nagio Takigawa, Katsuyuki Kiura. The Role of STAT3 in Non-small cell lung cancer. Cancer. 2014; 6:708-22.

[34] MA Hong, PENG Zhangzhe, HU Gaoyun et al. Effects of fluorofenidone on organ fibrosis and the mechanisms. Journal of Central South University. Medical Science, 2015, 40(2): 208-213. 
[35] Sandro Goruppi, Juan Lucio Iovanna. Stress-inducible Protein p8 Is Involved in Several Physiological and Pathological Processes. J. Biol. Chem. 2010, 285:1577-1581.

[36] Vasseur S, Hoffmeister A, Garcia-Montero A et al. Mice with targeted disruption of $\mathrm{p} 8$ gene show increased sensitivity to lipopolysaccharide and DNA microarray analysis of livers reveals an aberrant gene expression response. BMC Gastroenterol. 2003, 3:25.

[37] Taïeb D, Malicet C, Garcia S et al. Inactivation of stress protein p8 increases murine carbon tetrachloride hepatotoxicity via preserved CYP2E1 activity. Hepatology. 2005, 42(1): 176-82.

[38] Cano CE, Hamidi T, Sandi MJ et al. Nupr1: the Swiss-knife of cancer. J Cell Physiol. 2011, 226(6): 1439-43.

[39] Hamidi T, Algül H, Cano CE et al. Nuclear protein 1 promotes pancreatic cancer development and protects cells from stress by inhibiting apoptosis. J Clin Invest.2012, 122(6):2092-103

[40] Guo X, Wang W, Hu J et al. Lentivirus-mediated RNAi knockdown of NUPR1 inhibits human nonsmall cell lung cancer growth in vitro and in vivo. Anat Rec (Hoboken). 2012, 295(12):2114-21.

[41] Pommier RM, Gout J, Vincent DF et al. The human NUPR1/P8 gene is transcriptionally activated by transforming growth factor $\beta$ via the SMAD signalling pathway. Biochem J. 2012, 445(2):285-93. 